\title{
AN UPPER LIMIT ON SUNSPOT ACTIVITY DURING THE MAUNDER MINIMUM
}

\author{
G. A. KOVALTSOV ${ }^{1}$, I. G. USOSKIN ${ }^{2}$ and K. MURSULA ${ }^{3}$ \\ ${ }^{1}$ Ioffe Physical-Technical Institute, 194021 St. Petersburg, Russia \\ ${ }^{2}$ Sodankylä Geophysical Observatory (Oulu unit), P.O.Box 3000, FIN-90014 University \\ of Oulu, Finland \\ (e-mail: Ilya.Usoskin@oulu.fi) \\ ${ }^{3}$ Department of Physical Sciences, P.O.Box 3000, FIN-90014 University of Oulu, Finland \\ (e-mail: kalevi.mursula@oulu.fi)
}

(Received 6 September 2004; accepted 30 September 2004)

\begin{abstract}
We have estimated the upper and lower limits of sunspot activity, in terms of active day fraction during the Maunder minimum (1645-1710), using raw information on individual daily observations (Hoyt and Schatten, 1998). Establishing the relation between the sunspot activity and active day fraction after 1850, we evaluate the upper limit of annual group sunspot number during the deep Maunder minimum (1645-1700) which does not exceed 4. The earlier finding of a dominant 22-year periodicity during the Maunder minimum is verified and shown to be robust. Also we confirm that the start of the Maunder minimum was very abrupt.
\end{abstract}

\section{Introduction}

Maunder minimum (MM) in 1645-1715 was a period when solar activity was drastically reduced for several decades. After a long debate, this fact was doubtlessly verified by Eddy (1976) using a careful analysis of different data sets. However, the quantitative level of solar activity remains yet a subject of debate. e.g., recent reconstructions of solar activity (Nagovitsyn, 1997; Rigozo et al., 2001) yield peak annual sunspot numbers of about 30 during the MM, which is in agreement with estimates by Schove (1955) but contradicts values suggested by Eddy (1976). A new series of group sunspot numbers (GSNs) was recently developed by Hoyt and Schatten (1998) increasing the set of observations by $80 \%$ compared to the Wolf series. This index of sunspot activity is more homogeneous and reliable than the traditional Wolf sunspot number (Hoyt, Schatten, and NesmesRibes, 1994; Hoyt and Schatten, 1998; Letfus, 1999). The group sunspot number series suggests that sunspot activity remained at an extremely low level during the MM (see, e.g., Wang and Sheeley, 2003), even lower than suggested by Eddy (1976).

A large fraction of the spotless days during MM are based on general statements on the absence of sunspots during a long period (year or longer). On the other hand, the annual percentage of those spotless days for which an explicit observational record exists, varies between 10 and $80 \%$. Although general statements were made mostly by professional astronomers (e.g., Hevelius and Picard), one cannot be $100 \%$ sure that all days were really observed during such periods. This was the reason for 
some criticism of the results obtained using group sunspot number series for the MM (e.g., Ogurtsov, Kocharov, and Nagovitsyn, 2003).

In this paper we try to evaluate the significance of such general statements and estimate an upper limit of sunspot activity during MM using only explicit daily observations collected by Hoyt and Schatten (1998).

\section{Data and Assumptions}

We analyze the statistics of active/total days of sunspot observations during the MM. We will compare two extreme hypothesis. One (called "Formal") is based on formal GSN daily values (Hoyt and Schatten, 1998). Besides actual daily observational reports, the series includes also general spotless statements about the absence of sunspot during a long period. Such general statements are regarded formally as actually observed spotless days. The series also contains interpolation of small gaps (e.g., a gap of a few days between two spotless days is filled by zeros).

We consider also a more conservative hypothesis (called "Explicit" henceforth) which is based upon the database of actual daily observations by individual observers (http://www.ngdc.noaa.gov/stp/sunspot_numbers/group_sunspot_numbers/ alldata.dat). In the framework of this hypothesis we consider only those days when at least one observer explicitly reported an observation. The day was called active if at least one observer reported sunspot activity. We did not fill small gaps by interpolations. General spotless statements were not used after 1665 when there were enough explicit observations. Since the number of explicit observations was small at the beginning of the MM and only general statements exist for the period of 1646-1651, we took these general statements into account but in a rather conservative manner. All general statements on long spotless periods (one year or longer) are substituted by only 24 spotless days evenly distributed within the year (cf. 365 days in the "Formal" hypothesis). This was done for the years 1645-1651 (general spotless statements by Hevelius) and 1653-1665 (general spotless statements by Picard). We note that 24 observational days per year correspond to about $50 \%$ and $25 \%$ of the averaged annual number of actual daily observations during the MM reported by Hevelius and Picard, respectively (Hoyt and Schatten, 1996).

The total number of observational days and active days used in the two hypotheses is shown in Figure 1. One can see that substituting all days covered by such general statements by spotless days as done by Hoyt and Schatten (1998) (dotted "Formal" curve in Figure 1) may, strictly speaking, lead to an underestimate of the actual sunspot activity level during the MM.

\section{Fraction of Active Days}

The fraction of active days (number $N_{\mathrm{a}}$ of days with at least one sunspot reported divided by the total number of observed days $N) F_{\mathrm{a}}=N_{\mathrm{a}} / N$ is taken 

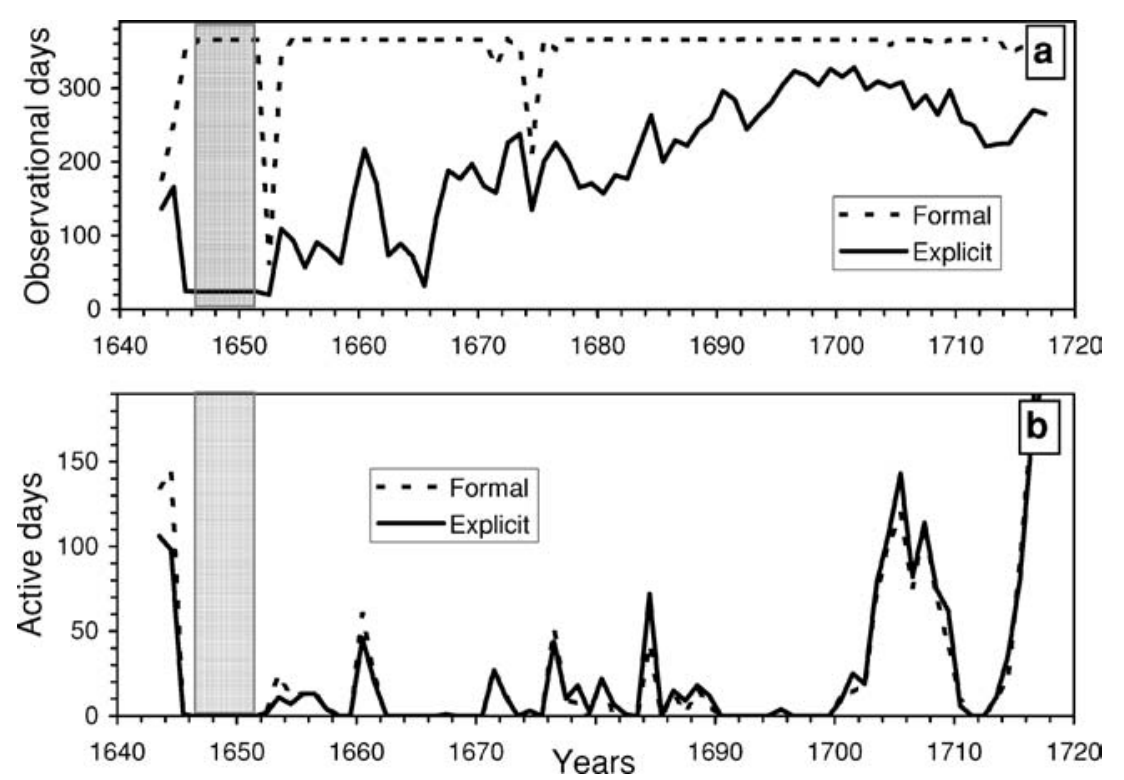

Figure 1. The number of observational days (panel a) and of active days (panel b) during the MM, according to Hoyt and Schatten (1998). Dotted "Formal" and solid "Explicit" curves correspond to the two used hypotheses (see text). The shaded area denotes years where only general statements exist.

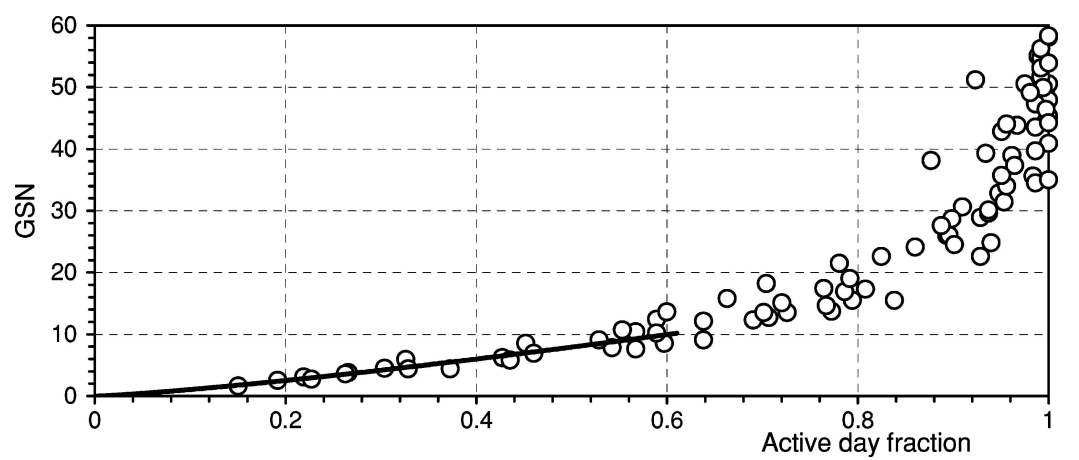

Figure 2. The group sunspot number as a function of the fraction of active days for the period 1850-2000. The solid line depicts a power-law approximation for GSN $<10\left(F_{\mathrm{a}}<0.6\right)$.

as a reliable indicator of sunspot activity during minimum times (Maunder, 1922; Harvey and White, 1999; Usoskin, Mursula, and Kovaltsov, 2000, 2001). Figure 2 shows the relation between the annual $F_{\mathrm{a}}$ and annual GSN for the period of reliable sunspot observations in 1850-2000. One can see that the fraction of active days $F_{\mathrm{a}}$ is non-ambiguously related to the GSN when the activity is low $\left(\mathrm{GSN}<10 ; F_{\mathrm{a}}<0.6\right)$. This implies that the low sunspot numbers are determined by the frequency of sunspot group occurrence rather than 
the number of simultaneously existing groups. For low sunspot activity, up to about GSN $<10\left(F_{\mathrm{a}}<0.6\right)$, the relation can be approximated by a power law

$$
\mathrm{GSN}=19 \times F_{\mathrm{a}}^{1.25}
$$

However, medium or high sunspot activity cannot be adequately represented by the fraction of active days since the relation between GSN and $F_{\mathrm{a}}$ (Figure 2) becomes ambiguous and finally reaches saturation for GSN $>30$. This means that during medium and high sunspot activity the number of simultaneously existing groups, not the number of active days, determines the GSN values. Accordingly, the relation (1) can be applied only for the times of low sunspot activity, GSN $<10$ and $F_{\mathrm{a}}<0.6$.

Actually we know the number of active days $r$ out of $n$ observational days within a year. The mathematical expectation of $F_{\mathrm{a}}$ is then simply $r / n$. We would also like to know the uncertainties of $F_{\mathrm{a}}$ since the smaller $n$ is, the less precisely we are able to estimate $F_{\text {a }}$. This is equivalent to a standard probability problem ${ }^{1}$ : from a box containing $N$ balls (black or white) $n$ balls are taken randomly, $r$ of which are black. How many black balls are there in the box in total? This problem leads to the hyper-geometrical probability distribution:

$$
p(s)=\frac{s !(N-s) !}{(s-r) !(N-s-n+r) !} \cdot \frac{n !(N-n) !}{(n-r) ! N ! r !}
$$

where $N=365$ or 366 (number of days in a year), and $s$ is the total number of active days within the year which needs to be estimated. Using the hyper-geometrical distribution, we have evaluated the mathematical expectation of $F_{\mathrm{a}}$ (the most probable value of $s$ ) together with the $90 \%$ (one-sided) significance level (s.l.). These are shown in Figure 3 for the two considered hypotheses. While the $90 \%$ s.l. intervals in the "Formal" hypothesis are narrow because of the larger effective number of observed days, they are wider for the "Explicit" hypothesis especially for 1645-1665 when there are many general statements and few explicit observations.

From Figure 3 we can obtain the uncertainty of the active day fraction during the Maunder minimum. The results are shown in Figure 4a, where the upper solid curve (upper limit of $F_{\mathrm{a}}$ ) corresponds to the upper $90 \%$ s.l. bound of the "Explicit" hypothesis (upper dotted curve in Figure 3a), and the lower curve to the lower bound of the "Formal" hypothesis (lower dotted curve in Figure 3b). Apparent are long spotless periods during 1662-1670 and 1690-1699. Applying now the regression given in Equation 1 we can estimate the corresponding upper and lower limits to the group sunspot number during the MM which are shown in Figure 4b.

\footnotetext{
${ }^{1}$ We implicitly assume here that sunspot observations are random and independent of each other. This is not exactly true since observations were usually more intense during days with sunspots. Accordingly, such an assumption yields a slightly overestimated $F_{\mathrm{a}}$ and GSN during the MM.
} 

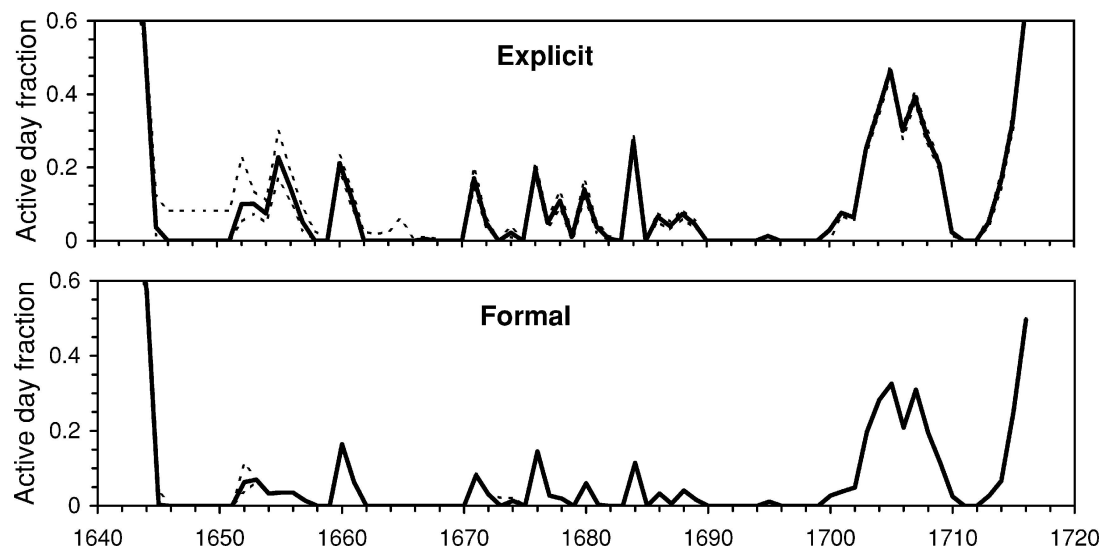

Figure 3. Mean (solid lines) and $90 \%$ significance level intervals (dotted lines) of the active day fraction for the two hypotheses, calculated for the Maunder minimum.
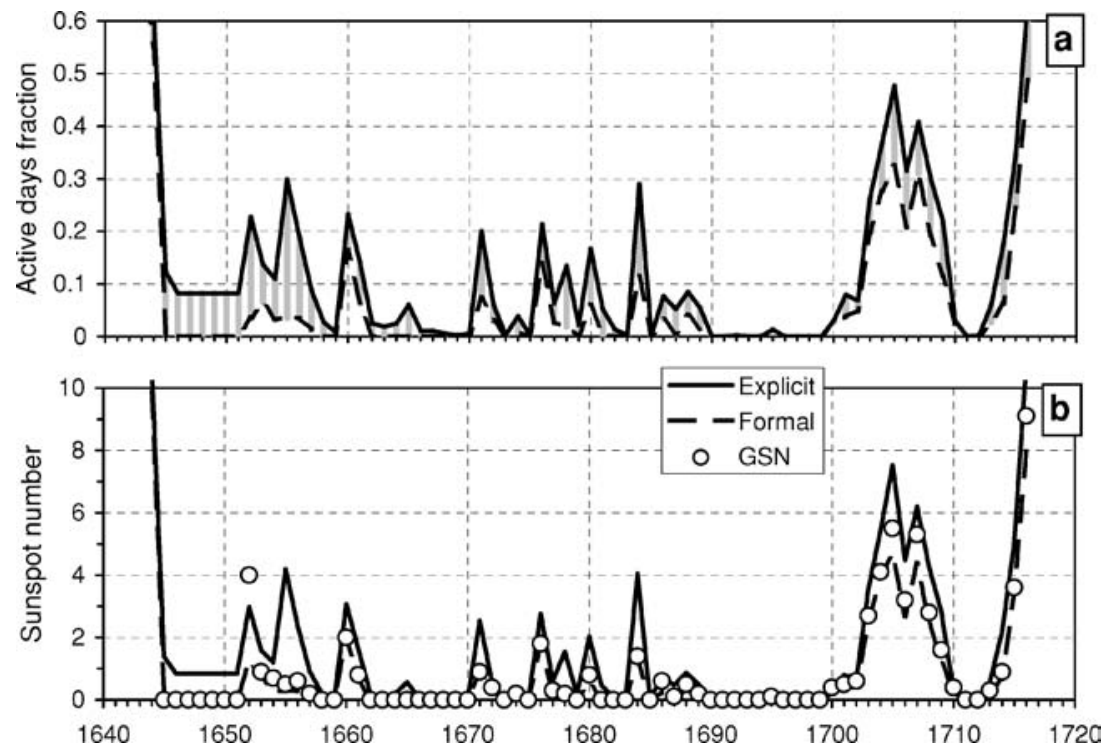

Figure 4. The intervals of uncertainties of sunspot activity during the Maunder minimum given as the upper $90 \%$ s.l. bound of the "Explicit" (solid curve) and lower $90 \%$ bound of the "Formal" hypotheses. (a) Active day fraction. (b) Sunspot numbers, dots depict the annual GSN values (Hoyt and Schatten, 1998).

\section{Discussion}

The formal annual GSN values by Hoyt and Schatten (1998) are shown by big dots in Figure $4 \mathrm{~b}$. These values were obtained using a procedure which is slightly different from the one used here. While Hoyt and Schatten (1998) calculated the 
annual GSN value in two steps - first calculating monthly values from daily sunspot numbers and then averaging them, we averaged all daily observations over the year. This procedure difference is responsible for the discrepancy (the GSN value lies above the "Explicit" curve) for the year 1652 with unevenly distributed individual daily observations. Nevertheless, the formal GSN annual values lie between the two of our estimates and closer to the "Formal" hypothesis (which is nearly identical to that used by Hoyt and Schatten, 1998). Such an agreement between the two approaches validates the use of the active day fraction as a reliable index of sunspot activity during low activity times.

Even in the "Explicit" hypothesis the upper limit of annual GSN values does not exceed 4 during 1645-1700. (Note that the "Explicit" curve further overestimates the sunspot activity by assuming that individual observations were independent (see footnote in Section 3), which makes it even more conservative.) Therefore, the very low level of sunspot activity (lower than the traditional Wolf numbers) during the MM is robust and is not affected, e.g., by the general (non-explicit) statements of long spotless periods in the beginning of the Maunder minimum. The solar cycle in 1700-1710 was also very tiny and the estimated annual GSN values did not exceed 8 (see Figure $4 \mathrm{~b}$ ), in agreement with the conclusion on the overestimation of sunspot activity level during the first half of 18 th century by Wolf (see, e.g., Letfus, 2000). One can see from Figure 4 long spotless periods during 1662-1670 and 1690-1699 and two periods of clustering of sunspot occurrence in 1652-1661 and 1671-1688, indicating a dominant 22-year cyclicity during the MM (Usoskin, Mursula, and Kovaltsov, 2000, 2001). This also confirms the very abrupt start of the Maunder minimum (Usoskin and Mursula, 2003).

Concluding, we have confirmed the very low level of sunspot activity during the Maunder minimum, using quite a conservative consideration of the data on direct sunspot observations. This should be considered as a test for models reconstructing sunspot activity from indirect proxies in the past.

\section{Acknowledgements}

We thank the Academy of Finland for financial support. GAK was partly supported by the program "Non-stationary processes in Astronomy" of the Russian Academy of Sciences.

\section{References}

Eddy, J. A.: 1976, Science 192, 1189.

Harvey, K. L. and White, O. R.: 1999, J. Geophys. Res. 104(A9), 19759.

Hoyt, D. V. and Schatten, K.: 1996, Solar Phys. 165, 181.

Hoyt, D. V. and Schatten, K.: 1998, Solar Phys. 179, 189.

Hoyt, D. V., Schatten, K. H., and Nesmes-Ribes, E.: 1994, Geophys. Res. Lett. 21, 2067. 
Letfus, V.: 1999, Solar Phys. 184, 201.

Letfus, V.: 2000, Solar Phys. 194, 175.

Maunder, E. W.: 1922, MNRAS, 82, 534.

Nagovitsyn, Yu. A.: 1997, Astron. Lett. 23, 742.

Ogurtsov, M. G., Kocharov, G. E., and Nagovitsyn, Yu. A.: 2003, Astron. Rep. 47, 517.

Rigozo, N. R., Echer, E., Vieira, L. E., and Nordemann, D. J. R.: 2001, Solar Phys. 203, 179.

Schove, D. J.: 1955, J. Geophys. Res. 60, 127.

Usoskin, I. G. and Mursula, K.: 2003, Solar Phys. 218, 319.

Usoskin, I. G., Mursula, K., and Kovaltsov, G. A.: 2000, Astron. Astrophys. 354.

Usoskin, I. G., Mursula, K., and Kovaltsov, G. A.: 2001, J. Geophys. Res. 106(A8), 16039.

Wang, Y.-M. and Sheeley, N. R., Jr.: 2003, Astrophys. J. 591, 1248. 